\title{
Inflationary predictions of double-well, Coleman-Weinberg, and hilltop potentials with non-minimal coupling
}

\author{
Nilay Bostan, Ömer Güleryüz and Vedat Nefer Şenoğuz* \\ Department of Physics, Mimar Sinan Fine Arts University, \\ 34380 Şişli, İstanbul, Turkey \\ E-mail: nilay.bostan@yeditepe.edu.tr, guleryuz17@itu.edu.tr, \\ nefer.senoguz@msgsu.edu.tr
}

\begin{abstract}
We discuss how the non-minimal coupling $\xi \phi^{2} R$ between the inflaton and the Ricci scalar affects the predictions of single field inflation models where the inflaton has a non-zero vacuum expectation value (VEV) $v$ after inflation. We show that, for inflaton values both above the VEV and below the VEV during inflation, under certain conditions the inflationary predictions become approximately the same as the predictions of the Starobinsky model. We then analyze inflation with double-well and Coleman-Weinberg potentials in detail, displaying the regions in the $v-\xi$ plane for which the spectral index $n_{s}$ and the tensor-to-scalar ratio $r$ values are compatible with the current observations. $r$ is always larger than 0.002 in these regions. Finally, we consider the effect of $\xi$ on small field inflation (hilltop) potentials.
\end{abstract}

Keywords: physics of the early universe, inflation

ArXiv ePrint: 1802.04160

\footnotetext{
${ }^{*}$ Corresponding author.
} 


\section{Contents}

1 Introduction 1

2 Inflation with non-minimal coupling $\quad 2$

2.1 Calculating the observational parameters 3

2.2 The Starobinsky conditions 6

3 Double-well potential $\quad 8$

4 Small field inflation potentials $\quad 11$

5 Coleman-Weinberg potential 13

6 Conclusion 16

\section{Introduction}

The hypothesis of cosmic inflation [1-4] provides a plausible explanation of the large scale homogeneity of the universe and, more importantly, of the primordial density perturbations that evolve into cosmic structure. A simple way inflation can occur is based on a slowrolling scalar field $\phi$ called the inflaton. Once the Lagrangian for the inflaton field and also the thermal history of the universe after inflation is specified, values for observational parameters can be calculated and compared with constraints coming from measurements of the cosmic microwave background (CMB) anisotropies [5, 6].

The observational parameters, in particular the scalar spectral index $n_{s}$ and the tensorto-scalar ratio $r$, have been calculated for various inflationary potentials (see [7] for a comprehensive subset). An assumption often made in the calculations is that the inflaton is minimally coupled. On the other hand, a renormalizable scalar field theory in curved spacetime also requires the non-minimal coupling $\xi \phi^{2} R$ between the inflaton and the Ricci scalar [8-10]. For a given potential, depending on the value of the non-minimal coupling parameter $\xi$, inflationary predictions and even whether inflation occurs or not can change [11-19].

Here we will investigate how the value of the non-minimal coupling parameter $\xi$ affects the inflationary predictions for potentials where the inflaton has a non-zero vacuum expectation value $(\mathrm{VEV}) v$ after inflation. In terms of the redefined field $\varphi \equiv \phi-v$, the non-minimal coupling in the Lagrangian includes a linear term in $\varphi$ as well as a quadratic term. Under some conditions on $\xi$ and $v$ that are discussed in section 2, this leads to inflationary predictions that approach those of the Starobinsky ( $R^{2}$ inflation) model [20], which is in good agreement with the current observations [6]. The Starobinsky-like behaviour is obtained not just for the well-known non-minimally coupled quartic potential case but also when inflation occurs near the quadratic minimum of the potential, for inflaton values above (below) the VEV and $\xi>0(\xi<0)$.

A reason for considering a non-zero VEV after inflation is that such potentials can be associated with symmetry breaking in the early universe. After a general discussion of inflation with non-minimal coupling for such potentials, we then analyze in detail two archetypal symmetry breaking potentials, namely the double-well potential (section 3) and 
the Coleman-Weinberg potential (section 5). Although both potentials with non-minimal coupling were previously considered, there are some gaps and disagreements in the literature which we address in these sections. For each potential, we display the observational parameter values as functions of $v$ for selected $\xi$ values as well as the regions in the $v-\xi$ plane for which the spectral index $n_{s}$ and tensor-to-scalar ratio $r$ values are compatible with the current observations. Section 4 suggests modifying the double-well potential to obtain a small field inflation (hilltop) potential, which unlike the other two potentials can fit observations for inflaton values below the VEV and $\xi, v \ll 1$. Finally, section 6 concludes the paper with a summary of our results and a remark on perturbative unitarity violation.

It is worth mentioning that we use the metric formulation of gravity throughout the paper. For inflation with a non-minimally coupled scalar field, the Palatini formulation leads to different predictions for cosmological parameters [21]. In particular, the attractor behaviour leading to the predictions of the Starobinsky model is lost, and $r$ can be much smaller compared to the metric formulation [21, 22].

\section{Inflation with non-minimal coupling}

Suppose we have a non-minimally coupled scalar field $\phi$ with a canonical kinetic term and a potential $V_{J}(\phi)$ :

$$
\frac{\mathcal{L}_{J}}{\sqrt{-g}}=\frac{1}{2} F(\phi) R-\frac{1}{2} g^{\mu \nu} \partial_{\mu} \phi \partial_{\nu} \phi-V_{J}(\phi),
$$

where the subscript $J$ indicates that the Lagrangian is specified in a Jordan frame. Here, for $V_{J}(\phi)$ we will be considering symmetry-breaking type of potentials where the inflaton $\phi$ takes positive values and has a non-zero vacuum expectation value (VEV) $v$ after inflation.

Our choice for $F(\phi)$ consists of a constant $m^{2}$ term and a non-minimal coupling $\xi \phi^{2} R$ between the inflaton and the Ricci scalar. The constant term is familiar from the EinsteinHilbert action, and the $\xi \phi^{2} R$ term is required in a renormalizable scalar field theory in curved space-time [8-10]. We are using units where the reduced Planck scale $m_{P}=1 / \sqrt{8 \pi G} \approx$ $2.4 \times 10^{18} \mathrm{GeV}$ is set equal to unity, so we require $F(\phi) \rightarrow 1$ after inflation. Therefore taking $m^{2}=1-\xi v^{2}$, we have $F(\phi)=m^{2}+\xi \phi^{2}=1+\xi\left(\phi^{2}-v^{2}\right)$. The $\xi v^{2}$ term in $F(\phi)$ can be neglected in some specific models such as when the standard model Higgs is the inflaton $[23,24]$, but may well play an important role in other models. For instance, if inflation is associated with symmetry breaking at or near the grand unified theory scale $v \sim 0.01$, $|\xi| v^{2} \gtrsim 1$ is possible for values of $|\xi| \gtrsim 10^{4}$ similar to values required for standard model Higgs inflation.

As we will see, as cosmological scales exit the horizon $F(\phi) \gtrsim 1$ in the observationally favored region of parameters, and the Starobinsky-like regime corresponds to $F(\phi) \gg 1$. The effective gravitational constant $G_{N}=1 /[8 \pi F(\phi)]$ remains positive throughout the evolution of the field. Indeed, if we switch to the Einstein frame and make a field redefinition so that the kinetic term is again canonical, we see that $G_{N}=0$ is only reached at infinite values of the field (see section 2.1 and ref. [25]). This implies, in particular, that if $\xi v^{2}>1$ there can be no transition from the symmetric $(\phi=0)$ phase to the broken-symmetry $(\phi=v)$ phase. Nevertheless, we include this case in our investigations as the field evolution does not have to start from the symmetric phase, and could for example start from values above the VEV as would be expected for chaotic initial conditions [4]. 
It has been appreciated $[16,25-33]$ that different choices for $F(\phi)$ and $V_{J}(\phi)$ can share the same attractor point with the Starobinsky ( $R^{2}$ inflation) model [20,34], which predicts

$$
n_{s}=1-\frac{2}{N}, \quad r=\frac{12}{N^{2}}, \quad \frac{\mathrm{d} n_{s}}{\mathrm{~d} \ln k}=-\frac{2}{N^{2}},
$$

to leading order in the number of e-folds $N$, where $\mathrm{d} n_{s} / \mathrm{d} \ln k$ is the running of the spectral index. An example relevant to our discussion is

$$
F(\phi)=1+\xi \phi^{n}, \quad V_{J}(\phi) \propto \phi^{2 n},
$$

which is a special case of the strong coupling attractor model discussed in [30] (see also [27]).

In terms of the redefined field $\varphi \equiv \phi-v$ so that $\varphi=0$ after inflation, $F(\varphi)=1+$ $\xi \varphi^{2}(1+2 v / \varphi)$ includes a linear term in $\varphi$ as well as a quadratic term. If $\phi^{2}-v^{2} \gg v^{2}$ $\left(\varphi^{2} \gg v^{2}\right)$ as cosmological scales exit the horizon, it means the inflaton is away from the minimum and $F(\varphi) \approx 1+\xi \varphi^{2}$. Then eq. (2.3) is satisfied for $V_{J}(\varphi) \propto \varphi^{4}$, the non-minimally coupled quartic model well-known since the late eighties $[15,16,35,36]$. On the other hand, if $\left|\phi^{2}-v^{2}\right| \ll v^{2}\left(\varphi^{2} \ll v^{2}\right)$ as cosmological scales exit the horizon, $F(\varphi) \approx 1+2 \xi v \varphi$ so that eq. (2.3) is satisfied for $V_{J}(\varphi) \propto \varphi^{2}$, with $\xi>0(\xi<0)$ for inflaton values above (below) the VEV during inflation.

Since a generic potential will be quadratic close enough to its minimum, it seems that a generic $V_{J}(\phi)$ can share the predictions of the Starobinsky model up to leading order in the number of e-folds $N$. For this to happen, $\xi$ and $v$ values should satisfy some constraints which we discuss in section 2.2. Before that, we briefly review how to calculate the observational parameters for inflation with non-minimal coupling.

\subsection{Calculating the observational parameters}

For calculating the observational parameters given eq. (2.1), it is convenient to switch to the Einstein $(E)$ frame by applying a Weyl rescaling $g_{\mu \nu}=\tilde{g}_{\mu \nu} / F(\phi)$, so that the Lagrangian density takes the form [37]

$$
\frac{\mathcal{L}_{E}}{\sqrt{-\tilde{g}}}=\frac{1}{2} \tilde{R}-\frac{1}{2 Z(\phi)} \tilde{g}^{\mu \nu} \partial_{\mu} \phi \partial_{\nu} \phi-V_{E}(\phi),
$$

where

$$
\frac{1}{Z(\phi)}=\frac{3}{2} \frac{F^{\prime}(\phi)^{2}}{F(\phi)^{2}}+\frac{1}{F(\phi)}, \quad V_{E}(\phi)=\frac{V_{J}(\phi)}{F(\phi)^{2}},
$$

and $F^{\prime} \equiv \mathrm{d} F / \mathrm{d} \phi$. If we make a field redefinition

$$
\mathrm{d} \sigma=\frac{\mathrm{d} \phi}{\sqrt{Z(\phi)}}
$$

we obtain the Lagrangian density for a minimally coupled scalar field $\sigma$ with a canonical kinetic term.

For $F(\phi)=1+\xi\left(\phi^{2}-v^{2}\right)$, eq. (2.5) gives

$$
\frac{1}{Z(\phi)}=\frac{1+\xi\left(\phi^{2}-v^{2}\right)+6 \xi^{2} \phi^{2}}{\left[1+\xi\left(\phi^{2}-v^{2}\right)\right]^{2}} .
$$

It will be useful to consider some simplifying cases of this expression: 
1. Weak coupling limit

If $\left|\xi\left(\phi^{2}-v^{2}\right)\right| \ll 1$ and $6 \xi^{2} \phi^{2} \ll 1, \phi \approx \sigma$ and $V_{J}(\phi) \approx V_{E}(\sigma)$. (Provided $|\xi| \ll$ $1 / 6$, these conditions will be satisfied when $|\xi| v^{2} \ll 1$ for inflation below the VEV, and $|\xi| \phi^{2} \ll 1$ for inflation above the VEV.) Then, the inflationary predictions are approximately the same as for minimal coupling in general. Note, however, that if $V_{J}(\phi)$ is very flat as cosmological scales exit the horizon, then even a small correction in the potential can significantly alter the inflationary predictions, as we will discuss in section 4 .

2. $\quad$ Induced gravity limit [38]

In this limit $\left(\xi v^{2}=1, F(\phi)=\xi \phi^{2}\right)$, eq. (2.7) simplifies to $Z(\phi)=\xi \phi^{2} /(1+6 \xi)$ and using eq. (2.6), we obtain

$$
\phi=v \exp \left(\sqrt{\frac{\xi}{1+6 \xi}} \sigma\right)
$$

where we took $\sigma(v)=0$.

3. $\quad$ Strong coupling limit

If $6 \xi^{2} \phi^{2} \gg\left|\xi\left(\phi^{2}-v^{2}\right)\right| \gg 1$, we have

$$
\frac{1}{Z(\phi)} \approx \frac{6 \phi^{2}}{\left(\phi^{2}-v^{2}\right)^{2}}
$$

Using eq. (2.6), we obtain $\phi^{2}-v^{2} \propto e^{2 \sigma / \sqrt{6}}$ where $\sigma$ is positive during inflation. This exponential behaviour in terms of the canonical field $\sigma$ makes it difficult to satisfy observations except for the special cases discussed in section 2.2 where the Einstein frame potential $V_{E}(\sigma)$ has a plateau due to cancellations between $V_{J}(\phi)$ and $F(\phi)^{2}$.

Once the Einstein frame potential is expressed in terms of the canonical $\sigma$ field, the observational parameters can be calculated using the slow-roll parameters (see ref. [39] for a review and references):

$$
\epsilon=\frac{1}{2}\left(\frac{V_{\sigma}}{V}\right)^{2}, \quad \eta=\frac{V_{\sigma \sigma}}{V}, \quad \xi^{2}=\frac{V_{\sigma} V_{\sigma \sigma \sigma}}{V^{2}},
$$

where $\sigma$ 's in the subscript denote derivatives. The spectral index $n_{s}$, the tensor-to-scalar ratio $r$ and the running of the spectral index $\mathrm{d} n_{s} / \mathrm{d} \ln k$ are given in the slow-roll approximation by

$$
n_{s}=1-6 \epsilon+2 \eta, \quad r=16 \epsilon, \quad \frac{\mathrm{d} n_{s}}{\mathrm{~d} \ln k}=16 \epsilon \eta-24 \epsilon^{2}-2 \xi^{2} .
$$

The amplitude of the curvature perturbation $\Delta_{\mathcal{R}}$ is given by

$$
\Delta_{\mathcal{R}}=\frac{1}{2 \sqrt{3} \pi} \frac{V^{3 / 2}}{\left|V_{\sigma}\right|},
$$

which should satisfy $\Delta_{\mathcal{R}}^{2} \approx 2.4 \times 10^{-9}$ from the Planck measurement [5] with the pivot scale chosen at $k_{*}=0.002 \mathrm{Mpc}^{-1}$. The number of e-folds is given by

$$
N_{*}=\int_{\sigma_{e}}^{\sigma_{*}} \frac{V \mathrm{~d} \sigma}{V_{\sigma}},
$$


where the subscript "*" denotes quantities when the scale corresponding to $k_{*}$ exited the horizon, and $\sigma_{e}$ is the inflaton value at the end of inflation, which we estimate by $\epsilon\left(\sigma_{e}\right)=1$.

Unfortunately, for general values of $\xi$ and $v$, it is difficult and inconvenient to express the potential in terms of the canonical field $\sigma$. We therefore rewrite these slow-roll expressions in terms of the original field $\phi$ for the numerical calculations, following the approach in ref. [25]. Using eq. (2.6), eq. (2.10) can be written as

$$
\epsilon=Z \epsilon_{\phi}, \quad \eta=Z \eta_{\phi}+\operatorname{sgn}\left(V^{\prime}\right) Z^{\prime} \sqrt{\frac{\epsilon_{\phi}}{2}}, \quad \xi^{2}=Z\left(Z \xi_{\phi}^{2}+3 \operatorname{sgn}\left(V^{\prime}\right) Z^{\prime} \eta_{\phi} \sqrt{\frac{\epsilon_{\phi}}{2}}+Z^{\prime \prime} \epsilon_{\phi}\right) .
$$

where we defined

$$
\epsilon_{\phi}=\frac{1}{2}\left(\frac{V^{\prime}}{V}\right)^{2}, \quad \eta_{\phi}=\frac{V^{\prime \prime}}{V}, \quad \xi_{\phi}^{2}=\frac{V^{\prime} V^{\prime \prime \prime}}{V^{2}} .
$$

Similarly, eq. (2.12) and eq. (2.13) can be written as

$$
\begin{aligned}
\Delta_{\mathcal{R}} & =\frac{1}{2 \sqrt{3} \pi} \frac{V^{3 / 2}}{\sqrt{Z}\left|V^{\prime}\right|}, \\
N_{*} & =\operatorname{sgn}\left(\mathrm{V}^{\prime}\right) \int_{\phi_{\mathrm{e}}}^{\phi_{*}} \frac{\mathrm{d} \phi}{\mathrm{Z}(\phi) \sqrt{2 \epsilon_{\phi}}} .
\end{aligned}
$$

To calculate the numerical values of $n_{s}, r$ and $\alpha$ we also need a numerical value of $N_{*}$. Assuming a standard thermal history after inflation,

$$
N_{*} \approx 64.7+\frac{1}{2} \ln \frac{\rho_{*}}{m_{P}^{4}}-\frac{1}{3\left(1+\omega_{r}\right)} \ln \frac{\rho_{e}}{m_{P}^{4}}+\left(\frac{1}{3\left(1+\omega_{r}\right)}-\frac{1}{4}\right) \ln \frac{\rho_{r}}{m_{P}^{4}} .
$$

Here $\rho_{e}=(3 / 2) V\left(\phi_{e}\right)$ is the energy density at the end of inflation, $\rho_{r}$ is the energy density at the end of reheating and $\omega_{r}$ is the equation of state parameter during reheating, which we take to be constant. ${ }^{1}$ Using eq. (2.12), we can express $\rho_{*}$ in terms of $r$ :

$$
\rho_{*} \approx V\left(\phi_{*}\right)=\frac{3 \pi^{2} \Delta_{\mathcal{R}}^{2} r}{2} .
$$

To represent a plausible range of $N_{*}$, we can consider three cases: In the high- $N$ case $\omega_{r}$ is taken to be $1 / 3$, which is equivalent to assuming instant reheating. In the middle- $N$ case we take $\omega_{r}=0$ and the reheat temperature $T_{r}=10^{9} \mathrm{GeV}$, calculating $\rho_{r}$ using the standard model value for the number of relativistic degrees of freedom $\left(g_{*}=106.75\right)$. In the low- $N$ case we take $T_{r}=100 \mathrm{GeV}$ (again with $\omega_{r}=0$ ). ${ }^{2}$ The $n_{s}$ vs. $r$ curve for each case is shown in figure 1 for the double-well potential (discussed in section 3 ) along with the $68 \%$ and $95 \%$ confidence level (CL) contours given by the Planck collaboration (Planck TT+lowP $+\mathrm{BKP}+$ lensing + ext) [5]. The figure shows that for the double-well potential, the fiducial $N_{*}$ values of 50 and 60 that are often used essentially coincide with the range expected from a standard thermal history after inflation. This is also the case for the Coleman-Weinberg potential discussed in section 5. However, $N_{*}$ is smaller (e.g. between approximately 45 and 55 if $v \sim 0.01$ ) for the

\footnotetext{
${ }^{1}$ For a derivation of eq. (2.18) see e.g. ref. [40]. Note that $N_{*}$ is defined in the Einstein frame. The number of e-folds in the Jordan frame $N_{*}^{J}=N_{*}+(1 / 2) \ln \left[F\left(\phi_{*}\right) / F\left(\phi_{e}\right)\right]$ can be noticeably different in the strong coupling limit [41-44]. However, in a Jordan frame calculation the additional term in $N_{*}^{J}$ would appear in both eqs. 2.17 and 2.18, leaving the physically observable quantities unchanged, as expected [45].

${ }^{2} T_{r}$ as low as $10 \mathrm{MeV}$ is consistent with big bang nucleosynthesis, however it is difficult to explain how baryogenesis could occur at such low temperatures.
} 


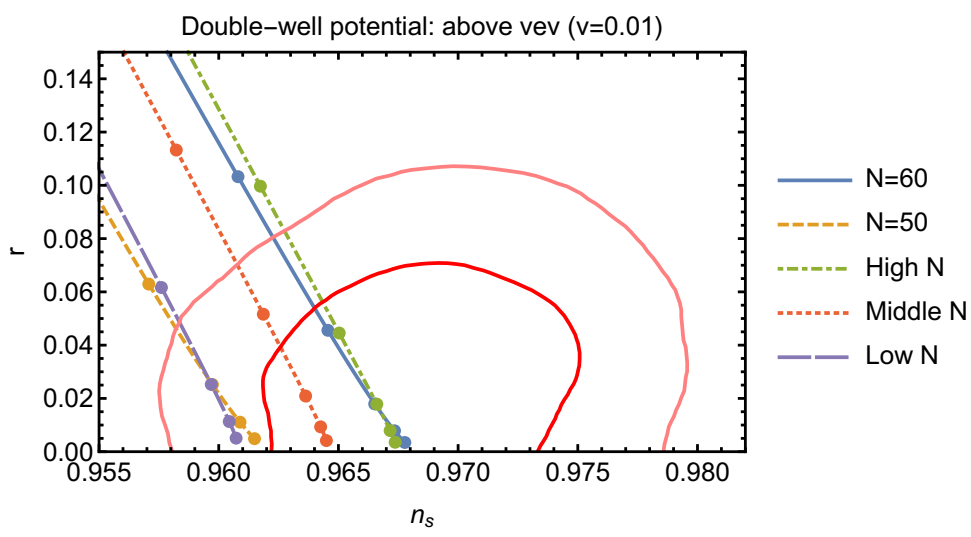

Figure 1. $n_{s}-r$ predictions for varying $\xi$ values and different reheating scenarios as explained in the text. The dots on the curves correspond to $\xi=10^{-2.5}, 10^{-2}, 10^{-1.5}, 0.1$, and 1 , top to bottom. The pink (red) contour corresponds to the $95 \%$ (68\%) CL contour given by the Planck collaboration (Planck TT+lowP+BKP+lensing+ext) [5].

small field inflation models discussed in section 4 due to inflation occurring at a lower energy scale.

We have carried out all the calculations in this article up to the leading order in the slow-roll parameters. Higher order corrections slightly change the values of the observational parameters (see e.g. refs. $[39,44]$ ). However, the uncertainty in the values of these parameters due to the reheating stage is much larger compared to the theoretical errors associated with the slow-roll approximation.

\subsection{The Starobinsky conditions}

As mentioned in the beginning of section 2, for a potential $V_{J}(\varphi)$ which is quartic away from the minimum or quadratic close to the minimum, if some conditions on $\xi$ and $v$ values are satisfied, predictions approach the Starobinsky point given by eq. (2.2) on the $n_{s}-r$ plane. Following the discussion in ref. [33], we will now derive these conditions using the relation of the Starobinsky point with the order and residue of the leading pole in the kinetic term.

Let's write the Einstein frame Lagrangian density in terms of $\chi(\phi) \equiv 1 / F(\phi)$ :

$$
\frac{\mathcal{L}_{E}}{\sqrt{-\tilde{g}}}=\frac{1}{2} \tilde{R}-\frac{1}{2} K(\chi) \tilde{g}^{\mu \nu} \partial_{\mu} \chi \partial_{\nu} \chi-V_{E}(\chi) .
$$

Suppose $K(\chi)$ is given by a Laurent series with a leading pole located at $\chi=0$ whereas $V_{E}(\chi)$ is given by a Taylor series starting from a non-vanishing constant term $U_{0}$ as cosmological scales exit the horizon:

$$
K(\chi)=\frac{a_{p}}{\chi^{p}}+\cdots, \quad V_{E}(\chi)=U_{0}(1-c \chi+\cdots) .
$$

In analogy with motion of a particle for $L=(1 / 2) m \dot{x}^{2}-V(x)$ and $m \rightarrow \infty$, slow-roll inflation occurs for $\chi \rightarrow 0$. We can calculate the inflationary predictions using the usual slow-roll expressions (see section 2.1) and $\mathrm{d} \sigma=\sqrt{K(\chi)} \mathrm{d} \chi$, obtaining [33]

$$
N_{*} \approx \frac{a_{p} \chi_{*}^{1-p}}{c(p-1)}, \quad n_{s} \approx 1-\frac{p}{(p-1) N_{*}}, \quad r \approx 8\left(\frac{c^{p-2} a_{p}}{\left[(p-1) N_{*}\right]^{p}}\right)^{\frac{1}{p-1}} .
$$


For a standard thermal history after inflation, the current data [5, 6] favors $n_{s} \approx 1-2 / N_{*}$, which corresponds to the case $p=2$. Note that for this case $r=8 a_{2} / N_{*}^{2}$ does not depend on $c$, which is to be expected since the kinetic term is invariant under $\chi \rightarrow c \chi$. The Starobinsky model predictions given by eq. (2.2) correspond to $p=2$ and $a_{2}=3 / 2$.

Now consider inflation with $F(\phi)=1+\xi\left(\phi^{2}-v^{2}\right)$, so that $\chi \rightarrow 0$ corresponds to $\xi\left(\phi^{2}-v^{2}\right) \gg 1$. This implies that we can look for Starobinsky-like solutions, with inflaton values above (below) the $\mathrm{VEV}$ if $\xi>0(\xi<0)$. Using eq. (2.5), we obtain

$$
K(\chi)=\frac{3}{2 \chi^{2}}+\frac{1}{4 \xi \chi^{2}\left[1-\chi\left(1-\xi v^{2}\right)\right]} .
$$

Note that this equation differs from eq. (22) of ref. [33] due to the $\xi v^{2}$ term in $F(\phi)$. As a consequence, the kinetic term can remain positive after as well as during inflation for both signs of $\xi$.

First, consider above VEV solutions satisfying $\phi^{2}-v^{2} \gg v^{2}$ as cosmological scales exit the horizon. In this case

$$
K(\chi) \approx \frac{3 \alpha}{2 \chi^{2}}, \text { where } \alpha \equiv 1+\frac{1}{6 \xi} \approx\left\{\begin{array}{c}
1 \text { if } \xi \gg \frac{1}{6}, \\
\frac{1}{6 \xi} \text { if } \xi \ll \frac{1}{6} .
\end{array}\right.
$$

Note that from eq. (2.22), $\chi_{*} \approx a_{2} /\left(c N_{*}\right)$ with $a_{2}=3 \alpha / 2$, so $\chi_{*} \ll 1$ corresponds to $\xi \gg 1 /\left(4 c N_{*}\right)$. Also, the assumption $\phi^{2}-v^{2} \gg v^{2}$ corresponds to $\xi v^{2} \ll 2 c N_{*} /(3 \alpha)$. When these conditions are satisfied, the leading order inflationary predictions coincide with those of the $\alpha$-attractor models [33, 46, 47], namely,

$$
n_{s}=1-\frac{2}{N_{*}}, \quad r=\frac{12 \alpha}{N_{*}^{2}} .
$$

Second, let's assume that $\left|\phi^{2}-v^{2}\right| \ll v^{2}$ as cosmological scales exit the horizon. Further assuming $6|\xi| v^{2} \gg\left|\phi^{2}-v^{2}\right|$, eq. (2.23) simplifies to $K(\chi) \approx 3 /\left(2 \chi^{2}\right)$, that is, $p=2$ and $a_{2}=3 / 2$. Therefore the Starobinsky model predictions given by eq. (2.2) are obtained for inflaton values both above and below the VEV whenever these two assumptions are satisfied. Using $\chi_{*} \approx 3 /\left(2 c N_{*}\right)$, the two assumptions correspond to $|\xi| v^{2} \gg\left(2 c N_{*}\right) / 3$ and $\xi^{2} v^{2} \gg c N_{*} / 9$, respectively.

As long as these conditions are satisfied and $V_{E}(\chi)$ is given by eq. (2.21), the inflationary predictions will match the Starobinsky model predictions up to leading order in $N_{*}$. From eq. (2.21) we obtain

$$
V_{J}(\phi) \approx U_{0} \xi^{2}\left(\phi^{2}-v^{2}\right)^{2}\left(1+\frac{2-c}{\xi\left(\phi^{2}-v^{2}\right)}+\cdots\right),
$$

which implies that regardless of the value of $c$ (as long as it is $\ll 1 / \chi$ ), Starobinsky-like solutions are obtained if $V_{J}(\phi)$ is approximately given by the double well potential for which $c=2$, as cosmological scales exit the horizon. Therefore in terms of $\varphi \equiv \phi-v$, the potentials satisfying eq. (2.21) can be written as

$$
V_{J}(\varphi) \propto \varphi^{4}\left(1+2 \frac{v}{\varphi}\right)^{2}
$$

This again shows that the Starobinsky point given by eq. (2.2) is obtained for the $\varphi^{4}$ potential away from the minimum $\left(\varphi^{2} \gg v^{2}\right)$, and the $\varphi^{2}$ potential near the minimum $\left(\varphi^{2} \ll v^{2}\right)$. 
From $\chi_{*} \approx 3 /\left(4 N_{*}\right)$ (for $\left.c=2\right)$, the value of $\varphi$ as cosmological scales exit the horizon is $\varphi_{*}^{2} \approx 4 N_{*} /(3 \xi)$ and $\left|\varphi_{*}\right| \approx 2 N_{*} /(3|\xi| v)$ for these two cases, respectively.

We can summarize the Starobinsky conditions as follows. The inflationary predictions for $V_{J}(\phi)$ coincide with the Starobinsky model predictions up to leading order in $N_{*}$ if:

1. The inflaton is above the VEV,$V_{J}(\phi)$ is quartic for $\phi^{2} \gg v^{2}, \xi v^{2} \ll 4 N_{*} / 3$ and $\xi \gg 1 / 6$.

(On the other hand, from eq. $(2.24), r \approx 2 /\left(\xi N_{*}^{2}\right)$ for $1 /\left(8 N_{*}\right) \ll \xi \ll 1 / 6$.)

2. The potential is quadratic around the minimum for $\varphi^{2} \ll v^{2}$ and

$$
\xi^{2} v^{2} \gg \frac{2 N_{*}}{9} \text { if }|\xi|<\frac{1}{6}, \quad|\xi| v^{2} \gg \frac{4 N_{*}}{3} \text { if }|\xi|>\frac{1}{6},
$$

where $\xi>0(\xi<0)$ for inflaton values above (below) the VEV. These conditions satisfy the strong coupling limit eq. (2.9), so that a plateau type Einstein frame potential is obtained during inflation in terms of the canonical scalar field:

$$
V_{E}(\sigma) \approx U_{0}\left(1-e^{-2 \sigma / \sqrt{6}}\right) .
$$

Both 1. and 2. are special cases of the strong coupling attractor model eq. (2.3), with $n=2$ and $n=1$, respectively. The $n=1$ case is discussed in ref. [31] and also belongs to the class of the induced inflation models discussed in ref. [32].

\section{Double-well potential}

In this section we analyze the prototypical symmetry breaking potential [48]

$$
V_{J}(\phi)=V_{0}\left[1-\left(\frac{\phi}{v}\right)^{2}\right]^{2},
$$

referred to as the double-well potential, the Higgs potential or the Landau-Ginzburg potential. First, let us briefly review inflation with this potential for the minimal coupling case, which was analyzed in several papers, see e.g. refs. [7, 49-56]. When inflation occurs near the minimum, in terms of $\varphi \equiv \phi-v$, the potential is approximately quadratic: $V \approx\left(4 V_{0} / v^{2}\right) \varphi^{2}$. Since $\varphi_{*}^{2} \approx 4 N_{*}$ for quadratic inflation, the observable part of inflation occurs near the minimum for $v^{2} \gg 4 N_{*}$. Then the quadratic potential predictions of

$$
n_{s} \approx 1-\frac{2}{N_{*}}, \quad r \approx \frac{8}{N_{*}}, \quad \frac{\mathrm{d} n_{s}}{\mathrm{~d} \ln k} \approx-\frac{2}{N^{2}},
$$

are obtained for inflation both below the VEV and above the VEV.

For inflation above the VEV, if $v^{2} \ll 4 N_{*}$ then we have quartic inflation with $n_{s} \approx 1-$ $3 / N_{*}$ and $r \approx 16 / N_{*}$. The predictions interpolate between the quadratic and quartic limits for $v^{2} \sim 4 N_{*}$, remaining out of the $95 \%$ CL Planck contour (Planck TT+lowP+BKP+lensing +ext) [5] for all $v$. Whereas for inflation below the VEV, if $v^{2} \ll 4 N_{*}$ then $\phi \ll v$ as cosmological scales exit the horizon, so the potential is effectively of the new inflation (small field or hilltop inflation) type

$$
V(\phi) \approx V_{0}\left[1-2\left(\frac{\phi}{v}\right)^{2}\right]
$$



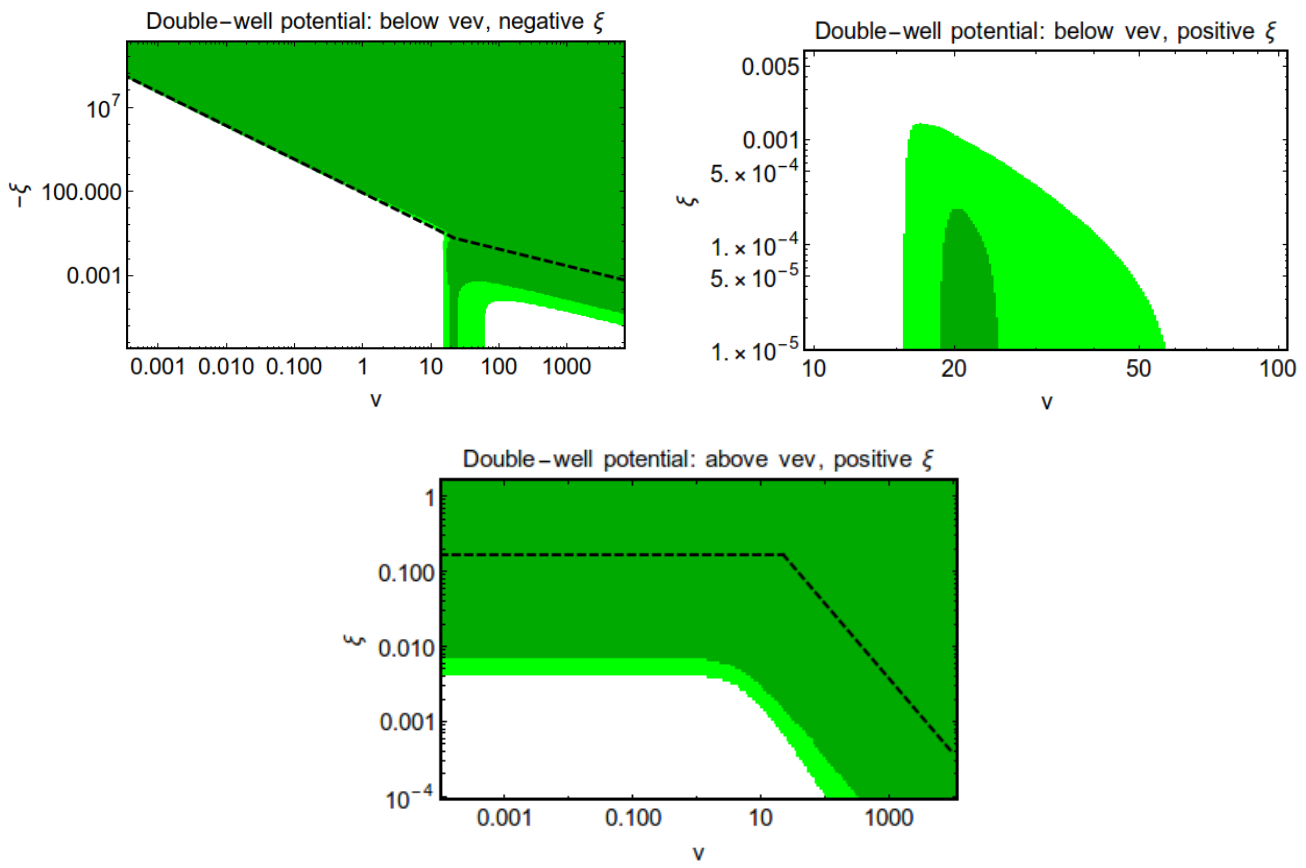

Figure 2. Light green (green) regions in the $v-\xi$ plane predict $n_{s}$ and $r$ values inside the $95 \%(68 \%)$ CL Planck contour (Planck TT+lowP+BKP+lensing+ext) [5]. The Starobinsky conditions eq. (2.28) and eq. (3.5) are satisfied above the dashed lines.

which implies a strongly red tilted spectrum $n_{s} \approx 1-8 / v^{2}$ with suppressed $r$. As a result, although both the $v^{2} \ll 4 N_{*}$ and $v^{2} \gg 4 N_{*}$ limits are ruled out, the $n_{s^{-}} r$ values are in the $68 \%$ CL Planck contour (Planck TT+lowP+BKP+lensing+ext) [5] for a narrow range around $v^{2} \sim 4 N_{*}$ (specifically, between $v=19$ and 25 for the high- $N$ case), see figures 2 and 3. Note that all the figures in this section are obtained for the high- $N$ case, using the equations given in section 2.1. In particular from eq. (2.17) we obtain:

$$
N_{*}=\frac{1}{8}(1+6 \xi)\left(\phi_{*}^{2}-\phi_{e}^{2}\right)+\frac{v^{2}}{4} \ln \frac{\phi_{e}}{\phi_{*}}+\frac{3}{4} \ln \frac{1+\xi\left(\phi_{e}^{2}-v^{2}\right)}{1+\xi\left(\phi_{*}^{2}-v^{2}\right)} .
$$

From the discussions in section 2, we expect that we need $\xi<0(\xi>0)$ to improve the fit to the observations for inflation below (above) the VEV. Indeed, figures 2 and 3 show that for inflation below the VEV, the predictions move out of the 95\% CL Planck contour for $\xi \gtrsim 10^{-3}$. For inflation above the VEV, the $\xi=0$ case interpolating between quadratic and quartic inflation is already out of the Planck range, as is the $\xi<0$ case which leads to an even redder spectrum and larger $r$.

It was discussed in section 2 that if certain constraints on $v$ and $\xi$ values are satisfied, a potential quadratic near its minimum or a potential quartic away from it correspond to special cases of the strong coupling attractor model of ref. [30]. The double-well potential satisfies both conditions. The Einstein frame potential can be written as

$$
V_{E}(\chi)=\frac{V_{0}}{\xi^{2} v^{4}}\left(1-2 \chi+\chi^{2}\right)
$$

Therefore the discussion in section 2.2 is directly applicable. In particular, eq. (2.28) implies that for inflation below the $\mathrm{VEV}$, as $|\xi|$ is increased for a given $v$, the predictions eventually 

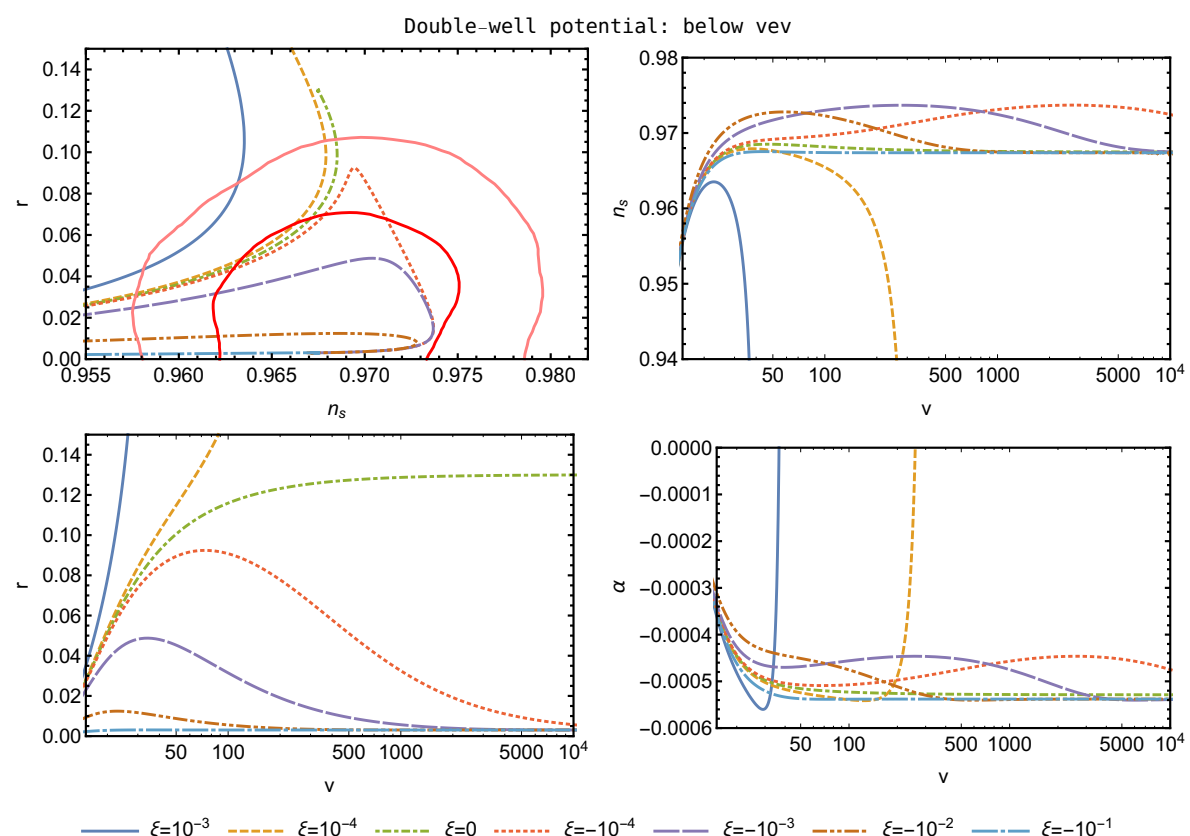

Figure 3. Observational parameter values as functions of $v$ for selected $\xi$ values. The pink (red) contour corresponds to the $95 \%(68 \%) \mathrm{CL}$ contour given by the Planck collaboration (Planck $\mathrm{TT}+$ lowP $+\mathrm{BKP}+$ lensing + ext) $[5]$.

approach the Starobinsky point given by eq. (2.2). As can be seen from figure 2, this transition is rather abrupt for $v^{2} \ll 8 N_{*}$ and occurs near $|\xi| v^{2}=4 N_{*} / 3$. This means that if the Starobinsky point is excluded by future observations, the entire parameter space $v^{2} \ll 8 N_{*}$ will be ruled out for below VEV inflation with this potential.

For above VEV inflation with $\xi v^{2} \ll 4 N_{*} / 3$ (which includes the induced gravity case $\left.\xi v^{2}=1\right)$ and $\xi \gg 1 /\left(8 N_{*}\right)$, the inflationary predictions are given by eq. (2.25). Thus, the $n_{s}-r$ values are in the $95 \%$ (68\%) CL contours for $\xi \gtrsim 0.004(0.007)$ for the high- $N$ case, see figures 2 and 4 . Note that for very low reheat temperatures the $n_{s}-r$ values can move out of the $68 \%$ CL contour, see figure 1 . For negligible values of $v$, as is the case in standard model Higgs inflation [23], the model is reduced to the non-minimally coupled quartic inflation model. Our results in this limit agree with previous results [15, 16, 35, 36].

Combining eq. (2.25) with eq. (2.28) we see that for above VEV inflation the Starobinsky point is obtained when:

$$
\xi^{2} v^{2} \gg \frac{2 N_{*}}{9} \text { if } 0<\xi<\frac{1}{6}, \quad \forall v \text { if } \xi \gg \frac{1}{6} .
$$

In the induced gravity limit, using eq. (2.8), the Einstein frame potential can be written as

$$
V_{E}(\sigma)=\frac{V_{0}}{\xi^{2} v^{4}}\left(1-\exp \left[\frac{-2 \sigma}{\sqrt{6 \alpha}}\right]\right)^{2}
$$

coinciding with the $\alpha$ - $\beta$ model of refs. [46, 47]. Thus the inflationary predictions approach the quadratic potential predictions given by eq. (3.2) for $\xi \ll 1 /\left(16 N_{*}\right)$, and eq. (2.25) for larger $\xi$. The double-well potential in the induced gravity limit was previously considered for inflation in refs. [13, 43, 57-59]. Ref. [58] also calculated $n_{s}$ for $\xi v^{2}$ values between 0 and 1. 

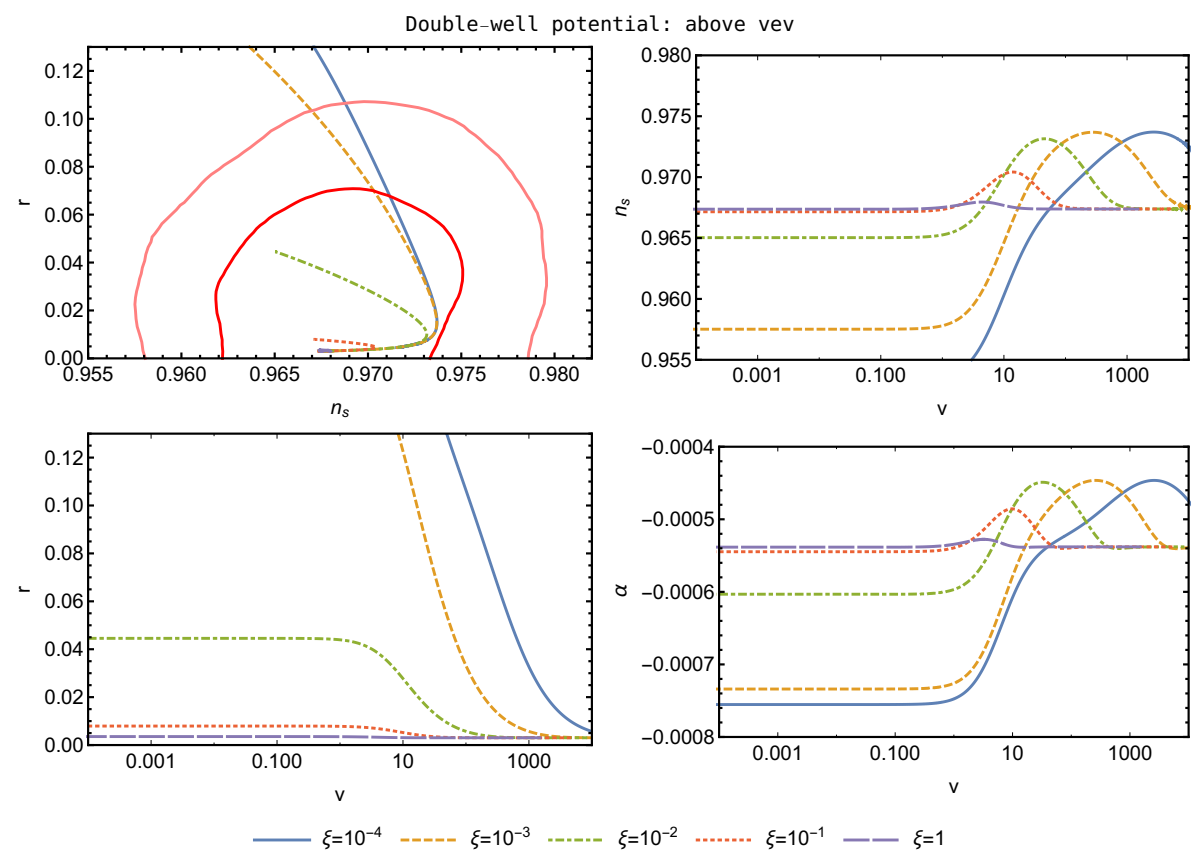

Figure 4. Observational parameter values as functions of $v$ for selected $\xi$ values. The pink (red) contour corresponds to the 95\% (68\%) CL contour given by the Planck collaboration (Planck $\mathrm{TT}+$ low $\mathrm{P}+\mathrm{BKP}+$ lensing + ext) $[5]$.

Our results agree with ref. [58] for $\xi v^{2} \ll 1$ but not for the induced gravity limit $\xi v^{2} \rightarrow 1$. For the latter case our results agree with refs. [43, 59].

Finally, ref. [25] analyzed the double-well potential with non-minimal coupling in detail (see also ref. [60] for the $\xi>0$ case). The difference between our work and theirs is that we take $F(\phi)=m^{2}+\xi \phi^{2}=1+\xi\left(\phi^{2}-v^{2}\right)$ as explained in section 2, whereas they take $F(\phi)=1+\xi \phi^{2}$. As a consequence, although the predictions on the $n_{s}-r$ plane look generally similar, there are a few differences between our and their results. Namely, for inflation below the VEV their predictions approach the Starobinsky point given by eq. (2.2) when $\xi v^{2} \rightarrow-1$, whereas our predictions approach it when eq. (2.28) is satisfied. For above VEV inflation, their predictions approach eq. (2.2) only for large values of $\xi$, whereas our predictions approach it when eq. (3.5) is satisfied.

\section{Small field inflation potentials}

Consider new inflation type models where the inflaton is below the VEV during inflation. For the double-well potential, we see that consistency with observations require $|\xi| v^{2} \gtrsim 4 N_{*} / 3$ so that very large $|\xi|$ values are needed for sub-Planckian values of the VEV $v$. On the other hand, a potential which is flatter near the origin could be compatible with observations even if $|\xi| \ll 1 / 6$. As an example we take a simple generalization of the double-well potential:

$$
V_{J}(\phi)=V_{0}\left[1-\left(\frac{\phi}{v}\right)^{p}\right]^{2}, \quad(p>2) .
$$



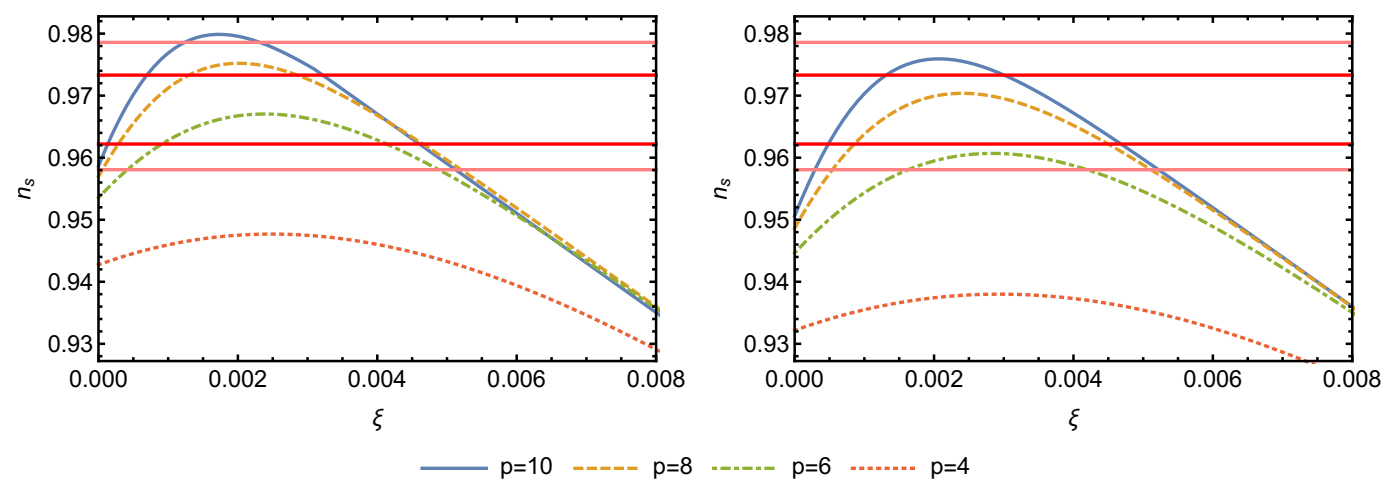

Figure 5. $n_{s}$ values as functions of $\xi$ for $\mu=0.01$ and selected $p$ values. Left panel: high- $N$ scenario, right panel: low- $N$ scenario (see section 2). The pink (red) line corresponds to the 95\% (68\%) CL contour given by the Planck collaboration (Planck TT+lowP+BKP+lensing+ext) [5].

For the weak coupling limit $|\xi| \ll 1 / 6$ and $|\xi| v^{2} \ll 1$, we have $\phi \approx \sigma$. If $v^{2} \ll 4 N_{*}$ then $\sigma \ll v$ during inflation, and the Einstein frame potential can be written as

$$
V_{E}(\sigma) \approx V_{0}\left[1-\left(\frac{\sigma}{\mu}\right)^{p}-2 \xi \sigma^{2}\right]
$$

where we have defined $\mu=v / 2^{1 / p}{ }^{3}$

For $\xi=0$, this small field inflation potential (also called hilltop potential) appears often in the literature, see for example refs. [7, 39, 67] and references therein. Using eqs. 2.10, 2.11 and 2.13 , we obtain

$$
n_{s} \approx 1-\frac{(p-1) 2}{(p-2) N_{*}}, \quad r \approx 128\left(\frac{16 \mu^{2 p}}{p^{2}\left[4(p-2) N_{*}\right]^{2 p-2}}\right)^{\frac{1}{p-2}},
$$

which shows that $r$ is suppressed and $n_{s}$ tends to be smaller than the range favored by observations. To be more specific, let's consider the most optimistic high- $N$ case, where using eqs. $2.18,2.19$, and $\rho_{*} \approx \rho_{e}$, we have

$$
N_{*} \approx 64.7+\frac{1}{4} \ln \frac{3 \pi^{2} \Delta_{\mathcal{R}}^{2} r}{2} .
$$

Note that the energy scale during inflation is lower for lower $\mu$ values, which correspond to lower $r, N_{*}$ and therefore $n_{s}$ values as well. For $\mu=0.01, n_{s}$ can be inside the $95 \% \mathrm{CL}$ contour given by the Planck collaboration (Planck TT+lowP+BKP+lensing+ext) [5] only for $p \geq 10$, see figure 5 . If $\mu \lesssim 6 \times 10^{-8}, n_{s}$ is outside the $95 \%$ CL contour for any $p$ value.

Repeating the calculation for the potential given by eq. (4.2), we obtain

$$
n_{s} \approx 1+\frac{8(p-1) \xi}{1-e^{4(p-2) \xi N_{*}}}-8 \xi, \quad r \approx \frac{128 \xi^{2} \mu^{2}\left(4 \xi \mu^{2} / p\right)^{2 /(p-2)} e^{8(p-2) \xi N_{*}}}{\left(e^{4(p-2) \xi N_{*}}-1\right)^{2(p-1) /(p-2)}} .
$$

These expressions are in excellent agreement with the numerical results given in figure 5, which were calculated using the Jordan frame potential given by eq. (4.1). They show that $n_{s}$ values increase and the fit to observational data improves provided $\xi \sim 1 /\left[4(p-2) N_{*}\right]$.

\footnotetext{
${ }^{3}$ This potential also arises in some supersymmetric new inflation models [61-64] and was analyzed in refs. $[65,66]$.
} 
In particular, $n_{s}$ can be inside the $95 \%$ CL contour for much smaller VEVs, namely for $\mu \gtrsim 2 \times 10^{-9}, 2 \times 10^{-17}, 7 \times 10^{-23}$ for $p=6,8,10$ respectively.

\section{Coleman-Weinberg potential}

Symmetry breaking due to the Coleman-Weinberg mechanism [68] was associated with inflation since the early eighties when the first new inflation models were proposed [2, 3, 69]. In these models the effective potential can be written as $[70,71]$

$$
V_{J}(\phi)=A \phi^{4}\left[\ln \left(\frac{\phi}{v}\right)-\frac{1}{4}\right]+\frac{A v^{4}}{4} .
$$

For a minimally coupled scalar, the inflationary predictions of this potential were analyzed in ref. [72] (see also refs. [7, 53-55, 73-75]). They are generally similar to the predictions of the double-well potential: Again, for $v^{2} \gg 4 N_{*}$ inflation occurs around the quadratic minimum $V \approx 2 A v^{2} \varphi^{2}$, leading to eq. (3.2). For inflation above the VEV, the predictions again interpolate between the quadratic and quartic limits, remaining out of the 95\% CL Planck contour. Whereas for inflation below the VEV, if $v^{2} \ll 4 N_{*}$ then $\phi \ll v$ as cosmological scales exit the horizon, so the potential is effectively of the new inflation (small field or hilltop inflation) type

$$
V(\phi) \approx V_{0}\left[1-\left(\frac{\phi}{\mu}\right)^{4}\right],
$$

which predicts $n_{s} \approx 1-3 / N_{*}, \alpha \approx-3 / N_{*}^{2}$ and a tiny $r$ as given by eq. (4.3). Comparing with eq. (5.1), the parameter $\mu$ in eq. (5.2) is given by

$$
\mu^{4} \approx-\frac{v^{4}}{4}\left(\ln \frac{\phi_{*}}{v}-\frac{1}{4}\right)^{-1}
$$

where using eq. (2.13) we obtain

$$
\left(\frac{\phi_{*}}{v}\right)^{2} \approx-\frac{v^{2}}{16 N_{*}}\left[W_{-1}\left(-\frac{v^{2}}{16 N_{*}}\right)\right]^{-1} .
$$

Here, $W_{-1}$ is a branch of the Lambert function satisfying $W(z) e^{W(z)}=z$.

Similarly to the double-well potential, although both the $v^{2} \ll 4 N_{*}$ and $v^{2} \gg 4 N_{*}$ limits are ruled out for inflation below the VEV, the $n_{s}-r$ values are in the $68 \%$ CL Planck contour (Planck TT+lowP+BKP+lensing +ext) [5] for a narrow range around $v^{2} \sim 4 N_{*}$ (specifically, between $v=20$ and 38 for the high- $N$ case), see figures 6 and 7 . Note that all the figures in this section are also obtained for the high- $N$ case.

From figure 6 we see that below VEV inflation predictions are incompatible with the observational data for $|\xi| \ll 1 / 6$ and $v^{2} \lesssim 1$. This is expected from the discussion in section 4 since under these conditions the Coleman-Weinberg potential is approximately given by eq. (4.2) with $p=4$ during inflation. As is clear from figure 5 and eq. (4.5), a small nonminimal coupling cannot bring $n_{s}$ into agreement with observations. For instance $n_{s}$ remains $\leq 0.945$ for $v=0.01$ even under the most favorable instant reheating assumption. This result agrees with refs. [76, 77], but disagrees with ref. [78] where the quartic term in eq. (4.2) is erroneously neglected. 

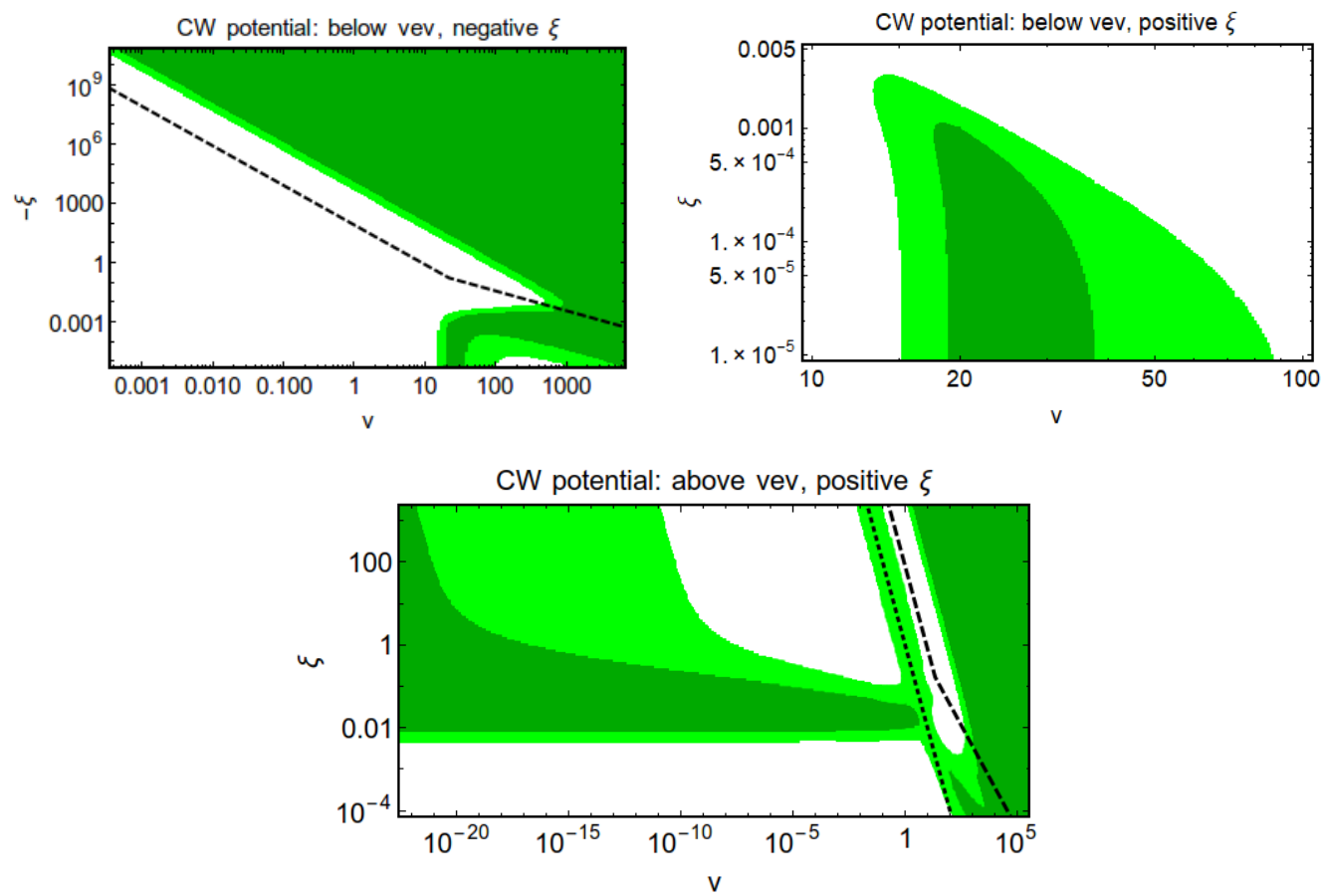

Figure 6. Light green (green) regions in the $v$ - $\xi$ plane predict $n_{s}$ and $r$ values inside the $95 \%(68 \%)$ CL Planck contour (Planck TT+lowP+BKP+lensing+ext) [5]. The Starobinsky conditions eq. (2.28) are satisfied above the dashed lines. The dotted line corresponds to $\xi v^{2}=1$.
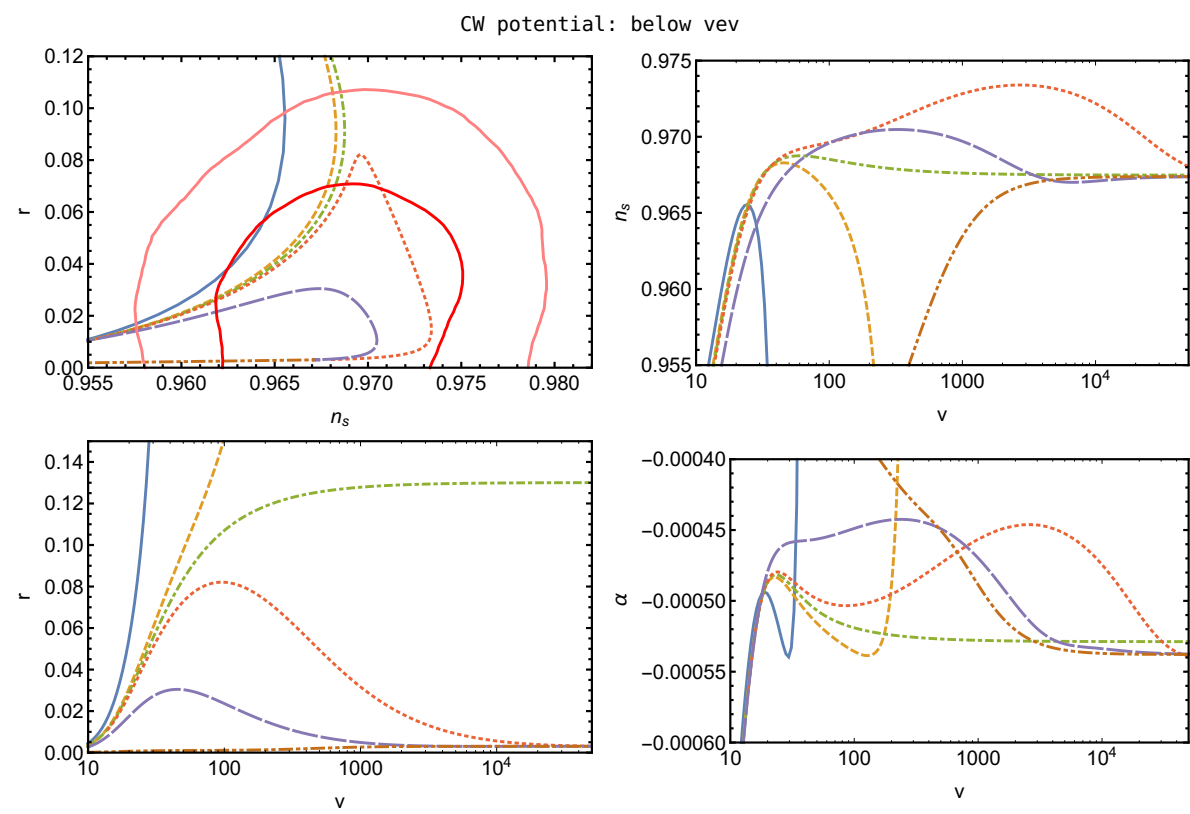

$\zeta=10^{-3}----\quad \xi=10^{-4}$

$\xi=0$

$\xi=-10^{-4}$

$\xi=-10^{-2}$

Figure 7. Observational parameter values as functions of $v$ for selected $\xi$ values. The pink (red) contour corresponds to the $95 \%$ (68\%) CL contour given by the Planck collaboration (Planck $\mathrm{TT}+$ lowP+BKP+lensing +ext) [5]. 

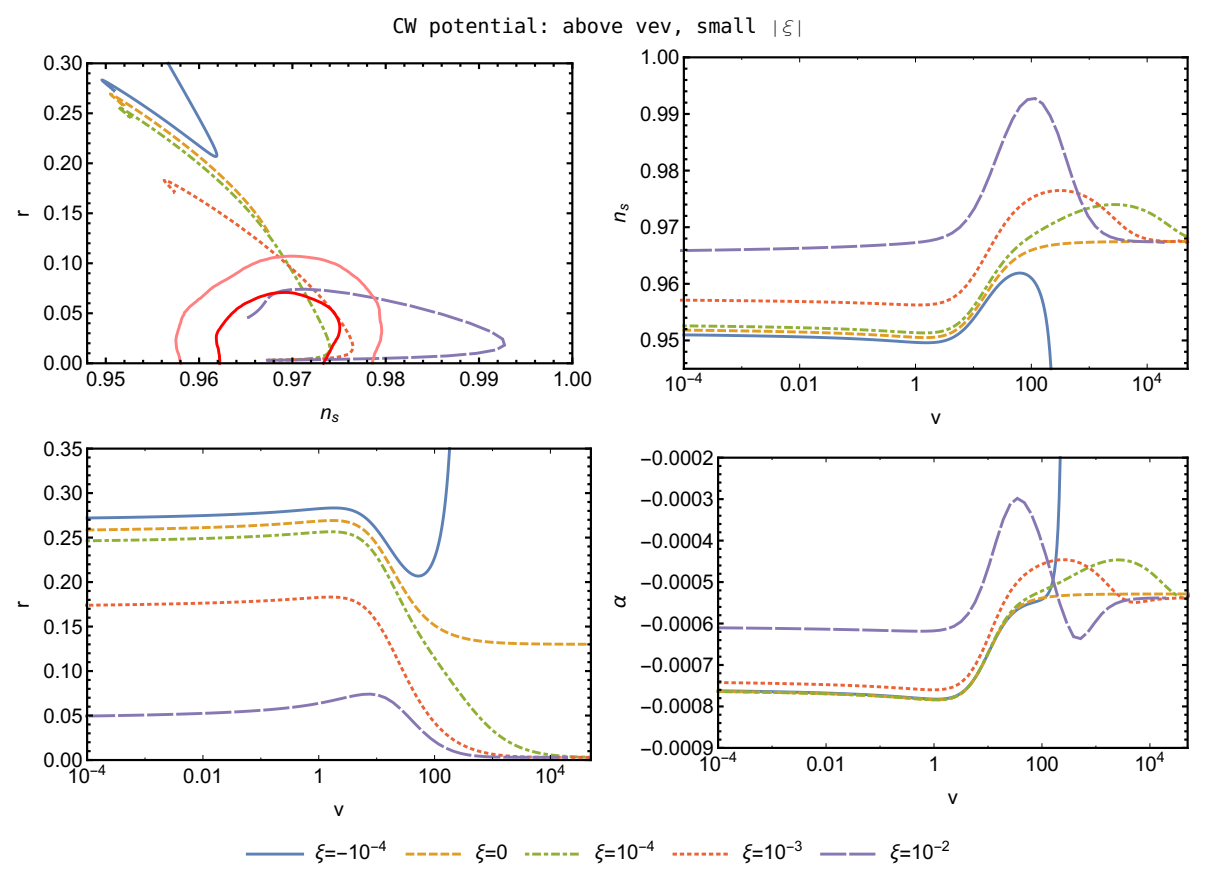

$\xi=-10^{-4} \ldots \ldots+\ldots=0, \ldots=10^{-4} \quad \ldots \ldots \ldots=10^{-3}$

Figure 8. Observational parameter values as functions of $v$ for selected $\xi$ values. The pink (red) contour corresponds to the $95 \%(68 \%) \mathrm{CL}$ contour given by the Planck collaboration (Planck $\mathrm{TT}+$ lowP+BKP+lensing +ext) [5].

The effect of the non-minimal coupling on the predictions of inflation below the VEV is similar for Coleman-Weinberg potential to the double-well potential. Both potentials are quadratic near their minima, so that the Starobinsky point given by eq. (2.2) is obtained when eq. (2.28) holds. Numerically, approaching the Starobinsky point requires even larger $|\xi| v^{2}$ for the Coleman-Weinberg potential as can be seen from figure 6 . The predictions move out of the $95 \%$ CL Planck contour for $\xi \gtrsim 3 \times 10^{-3}$.

For inflation above the VEV, the $\xi=0$ case interpolating between quadratic and quartic inflation is already out of the Planck range, as is the $\xi<0$ case which leads to an even redder spectrum and larger $r$ (see figure 8). The $\xi>0$ case, analyzed in ref. [79], is more subtle since the Coleman-Weinberg potential in the Jordan frame is not simply quartic away from the minimum but also contains a logarithmic factor. There is similarly a logarithmic factor in the Einstein frame potential written in terms of $\chi$ :

$$
V_{E}(\chi) \approx \frac{A}{2 \xi^{2}}\left[-\ln \left(\xi v^{2} \chi\right)\right](1-2 \chi) .
$$

Thus, the predictions of eq. (2.25) are approached only when the logarithmic factor can be treated as constant, that is, when the contribution from its derivative can be neglected. Taking derivative of eq. (5.5) and using $\chi_{*} \approx 3 \alpha /\left(4 N_{*}\right)$ (see section 2.2), we find that this requires

$$
\xi v^{2} \ll \frac{4 N_{*}}{3 \alpha} \exp \left[-\frac{2 N_{*}}{3 \alpha}\right] \text { for } \xi \gg \frac{1}{8 N_{*}} .
$$

Numerically, $n_{s^{-}} r$ values are in the $95 \%$ (68\%) CL contours for $\xi \gtrsim 0.005(0.008)$, assuming the high- $N$ case and $v \ll 1$, see figures 6 and 8. The Starobinsky point given by eq. $(2.2)$ 

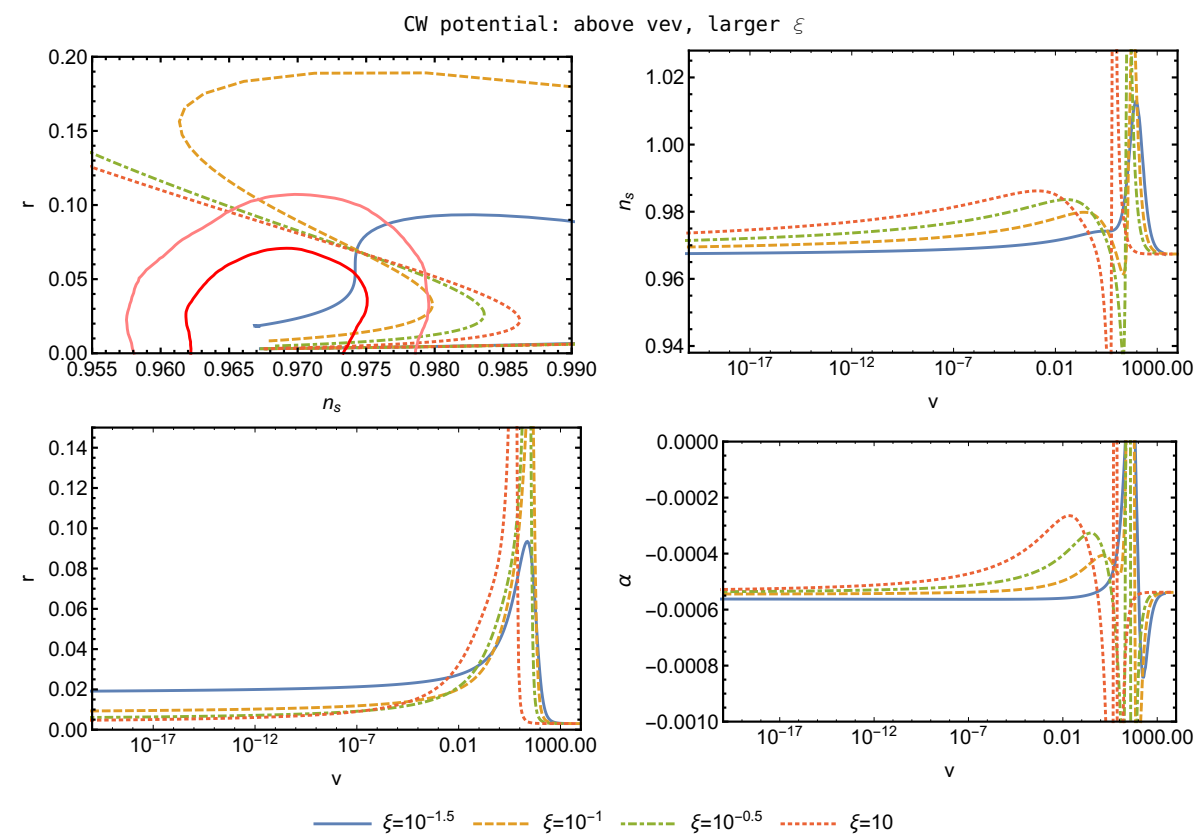

Figure 9. Observational parameter values as functions of $v$ for selected $\xi$ values. The pink (red) contour corresponds to the 95\% (68\%) CL contour given by the Planck collaboration (Planck $\mathrm{TT}+$ lowP $+\mathrm{BKP}+$ lensing + ext) $[5]$.

is obtained both for $\xi v^{2} \gg 4 N_{*} / 3$ and for $\xi \gg 1 / 6$ with extremely small values of $\xi v^{2}$, whereas the predictions move out of the observationally favored region in the $n_{s^{-}} r$ plane as $\xi v^{2}$ approaches $4 N_{*} / 3$ (see figure 9 ).

Using eq. (2.8) we can write the Einstein frame potential in the induced gravity limit $\left(\xi v^{2}=1\right)$ as follows [80]:

$$
V(\sigma)=\frac{A}{4 \xi^{2}}\left(4 \sqrt{\frac{\xi}{1+6 \xi}} \sigma+\exp \left(-4 \sqrt{\frac{\xi}{1+6 \xi}} \sigma\right)-1\right),
$$

where $\sigma>0(\sigma<0)$ during inflation above (below) the VEV. Analysis of this potential $[44,59,80]$ shows that inflation below the VEV is not compatible with the current observational data, whereas above VEV inflation predictions interpolate between the linear potential and quadratic potential predictions for $v^{2} \ll 2 N_{*}$ and $v^{2} \gg 2 N_{*}$, respectively. The linear potential predictions

$$
n_{s} \approx 1-\frac{3}{2 N_{*}}, \quad r \approx \frac{4}{N_{*}}, \quad \frac{\mathrm{d} n_{s}}{\mathrm{~d} \ln k} \approx-\frac{3}{2 N^{2}},
$$

are in the Planck \%95 CL contour, which explains the light green region around the dotted line in figure 6.

\section{Conclusion}

In this work we discussed the inflationary predictions of models with the Lagrangian

$$
\frac{\mathcal{L}_{J}}{\sqrt{-g}}=\frac{1}{2}\left(m^{2}+\xi \phi^{2}\right) R-\frac{1}{2} g^{\mu \nu} \partial_{\mu} \phi \partial_{\nu} \phi-V_{J}(\phi),
$$


where the inflaton $\phi$ has a non-zero VEV $v$ after inflation, and $m^{2}=1-\xi v^{2}$. In terms of the redefined field $\varphi \equiv \phi-v$, the non-minimal coupling in the Lagrangian includes a linear term in $\varphi$ as well as a quadratic term. This leads to an attractor behaviour where the predictions approach the Starobinsky model predictions, not just for the well-known nonminimally coupled quartic potential case but also when the inflaton is near the minimum $\left(\varphi^{2} \ll v^{2}\right)$ as cosmological scales exit the horizon.

After discussing the conditions under which Starobinsky-like behaviour is obtained in section 2, we analyze two prototypical symmetry breaking potentials: the double-well potential in section 3 and the Coleman-Weinberg potential in section 5. For each potential, we display the regions in the $v-\xi$ plane for which the spectral index $n_{s}$ and the tensor-to-scalar ratio $r$ values are compatible with the current observations.

If $\xi>0(\xi<0)$ for inflation above (below) the VEV, large portions of the $v-\xi$ plane lead to predictions compatible with the current constraints on $n_{s}$ and $r$, see figures 2 and 6 . Most of these portions lead to predictions approaching the Starobinsky model predictions, so the allowed parameter space would shrink drastically if future observations rule out the Starobinsky model. In particular, if the upper bound on $r$ becomes $<0.002$, both the double-well and Coleman-Weinberg potentials would be ruled out as inflationary models, for any value of $v$ and $\xi{ }^{4}$

Although we have displayed the inflationary predictions for a wide range of $v$ and $\xi$ values, it is questionable whether the entire range can be theoretically justified. In particular $v \gtrsim 1$ can be difficult to realize starting from a fundamental theory [81]. The value of $\xi$ is ambiguous unless the inflationary part of the Lagrangian is embedded in a specific theory (see e.g. $[19,82])$. However, for the well-known non-minimally coupled quartic potential solution, expanding the action around the vacuum reveals a cut-off scale $\Lambda=1 / \xi[27,83,84]$, and requiring this to be higher than the energy scale during inflation corresponds to $\xi \lesssim 300$ using eq. (2.19) and eq. (2.2). On the other hand, ref. [85] has emphasized that the cut-off scale depends on the background value of the field and can remain above the relevant energy scales during and after inflation. In any case consistency with observations only require $\xi \gtrsim 0.005$ for this solution.

Starobinsky-like inflationary predictions also arise when eq. (2.28) is satisfied. The observable part of inflation then occurs near the minimum where the potential is $\propto \varphi^{2}$, and $F(\varphi) \approx 1+2 \xi v \varphi$. This is another special case of the strong coupling attractor model [30]. Interestingly, even though the Starobinsky-like regime corresponds to $|\xi| v \gg 1$ (with $\xi>0$ and $\xi<0$ for inflation above and below the VEV, respectively), the cut-off remains at the Planck scale [31,32]. Thus, although consistency with observations require large values of $|\xi|$ for sub-Planckian VEVs, no perturbative unitarity violation is expected at scale $1 / \xi$ around the vacuum, unlike the non-minimally coupled quartic potential case. ${ }^{5}$

Finally, in section 4, we briefly considered a higher order version of the double-well potential, which for sub-Planckian VEVs corresponds to a small field (hilltop) inflation potential, with an additional quadratic term coming from the non-minimal coupling. Unlike the two above-mentioned potentials, for this potential inflation below a sub-Planckian VEV can be compatible with observations for a positive $\xi \lesssim 0.005$, and a tiny $r$ is predicted. All the

\footnotetext{
${ }^{4}$ All the results in this article is based on the metric formulation of gravity. If different approaches such as the Palatini formulation is used $r$ could be much smaller [21, 22].

${ }^{5}$ The cut-off scale around the vacuum changes when eq. (2.28) is satisfied since expanding the Einstein frame Lagrangian for small values of $\varphi$, the leading order kinetic term is no longer canonically normalized but instead given by $1+6 \xi^{2} v^{2} \gg 1$.
} 
considered potentials predict a running of the spectral index that is too small to be observed in the near future, with $\mathrm{d} n_{s} / \mathrm{d} \ln k$ typically around $-2 / N_{*}^{2}$.

\section{Acknowledgements}

VNŞ thanks Diederik Roest for a useful discussion. This work is supported by TÜBİTAK (The Scientific and Technological Research Council of Turkey) project number 116F385.

\section{References}

[1] A. H. Guth, The inflationary universe: A possible solution to the horizon and flatness problems, Phys. Rev. D23 (1981) 347.

[2] A. D. Linde, A new inflationary universe scenario: A possible solution of the horizon, flatness, homogeneity, isotropy and primordial monopole problems, Phys. Lett. 108B (1982) 389.

[3] A. Albrecht and P. J. Steinhardt, Cosmology for grand unified theories with radiatively induced symmetry breaking, Phys. Rev. Lett. 48 (1982) 1220.

[4] A. D. Linde, Chaotic inflation, Phys. Lett. 129B (1983) 177.

[5] Planck collaboration, P. A. R. Ade et al., Planck 2015 results. XIII. Cosmological parameters, Astron. Astrophys. 594 (2016) A13 [1502.01589].

[6] Planck collaboration, P. A. R. Ade et al., Planck 2015 results. XX. Constraints on inflation, Astron. Astrophys. 594 (2016) A20 [1502.02114].

[7] J. Martin, C. Ringeval and V. Vennin, Encyclopædia inflationaris, Phys. Dark Univ. 5-6 (2014) 75 [1303.3787].

[8] C. G. Callan, Jr., S. R. Coleman and R. Jackiw, A new improved energy - momentum tensor, Annals Phys. 59 (1970) 42.

[9] D. Z. Freedman and E. J. Weinberg, The energy-momentum tensor in scalar and gauge field theories, Annals Phys. 87 (1974) 354.

[10] I. L. Buchbinder, S. D. Odintsov and I. L. Shapiro, Effective action in quantum gravity. IOP Publishing Ltd, 1992.

[11] L. F. Abbott, Gravitational effects on the SU(5) breaking phase transition for a Coleman-Weinberg potential, Nucl. Phys. B185 (1981) 233.

[12] B. L. Spokoiny, Inflation and generation of perturbations in broken symmetric theory of gravity, Phys. Lett. 147B (1984) 39.

[13] F. Lucchin, S. Matarrese and M. D. Pollock, Inflation with a nonminimally coupled scalar field, Phys. Lett. 167B (1986) 163.

[14] T. Futamase and K.-i. Maeda, Chaotic inflationary scenario in models having nonminimal coupling with curvature, Phys. Rev. D39 (1989) 399.

[15] R. Fakir and W. G. Unruh, Improvement on cosmological chaotic inflation through nonminimal coupling, Phys. Rev. D41 (1990) 1783.

[16] D. S. Salopek, J. R. Bond and J. M. Bardeen, Designing density fluctuation spectra in inflation, Phys. Rev. D40 (1989) 1753.

[17] L. Amendola, M. Litterio and F. Occhionero, The phase space view of inflation. 1: The nonminimally coupled scalar field, Int. J. Mod. Phys. A5 (1990) 3861.

[18] V. Faraoni, Nonminimal coupling of the scalar field and inflation, Phys. Rev. D53 (1996) 6813 [astro-ph/9602111]. 
[19] V. Faraoni, Cosmology in scalar tensor gravity. Kluwer Academic Publishers, 2004.

[20] A. A. Starobinsky, A new type of isotropic cosmological models without singularity, Phys. Lett. 91B (1980) 99.

[21] F. Bauer and D. A. Demir, Inflation with non-minimal coupling: Metric versus Palatini formulations, Phys. Lett. B665 (2008) 222 [0803.2664].

[22] L. Järv, A. Racioppi and T. Tenkanen, Palatini side of inflationary attractors, Phys. Rev. D97 (2018) 083513 [1712.08471].

[23] F. L. Bezrukov and M. Shaposhnikov, The standard model Higgs boson as the inflaton, Phys. Lett. B659 (2008) 703 [0710.3755].

[24] M. Atkins and X. Calmet, Bounds on the nonminimal coupling of the Higgs boson to gravity, Phys. Rev. Lett. 110 (2013) 051301 [1211.0281].

[25] A. Linde, M. Noorbala and A. Westphal, Observational consequences of chaotic inflation with nonminimal coupling to gravity, JCAP 1103 (2011) 013 [1101.2652].

[26] B. Whitt, Fourth order gravity as general relativity plus matter, Phys. Lett. 145B (1984) 176.

[27] J. L. F. Barbon and J. R. Espinosa, On the naturalness of Higgs inflation, Phys. Rev. D79 (2009) 081302 [0903.0355].

[28] R. Kallosh and A. Linde, Universality class in conformal inflation, JCAP 1307 (2013) 002 [1306.5220].

[29] R. Kallosh and A. Linde, Non-minimal inflationary attractors, JCAP 1310 (2013) 033 [1307.7938].

[30] R. Kallosh, A. Linde and D. Roest, Universal attractor for inflation at strong coupling, Phys. Rev. Lett. 112 (2014) 011303 [1310.3950].

[31] A. Kehagias, A. Moradinezhad Dizgah and A. Riotto, Remarks on the Starobinsky model of inflation and its descendants, Phys. Rev. D89 (2014) 043527 [1312.1155].

[32] G. F. Giudice and H. M. Lee, Starobinsky-like inflation from induced gravity, Phys. Lett. B733 (2014) 58 [1402.2129].

[33] M. Galante, R. Kallosh, A. Linde and D. Roest, Unity of cosmological inflation attractors, Phys. Rev. Lett. 114 (2015) 141302 [1412.3797].

[34] V. F. Mukhanov and G. V. Chibisov, Quantum fluctuations and a nonsingular universe, JETP Lett. 33 (1981) 532.

[35] N. Okada, M. U. Rehman and Q. Shafi, Tensor to scalar ratio in non-minimal $\phi^{4}$ inflation, Phys. Rev. D82 (2010) 043502 [1005.5161].

[36] F. Bezrukov and D. Gorbunov, Light inflaton after LHC8 and WMAP9 results, JHEP 07 (2013) 140 [1303.4395].

[37] Y. Fujii and K. Maeda, The scalar-tensor theory of gravitation. Cambridge University Press, 2007.

[38] A. Zee, A broken symmetric theory of gravity, Phys. Rev. Lett. 42 (1979) 417.

[39] D. H. Lyth and A. R. Liddle, The primordial density perturbation: Cosmology, inflation and the origin of structure. Cambridge University Press, 2009.

[40] A. R. Liddle and S. M. Leach, How long before the end of inflation were observable perturbations produced?, Phys. Rev. D68 (2003) 103503 [astro-ph/0305263].

[41] R. N. Lerner and J. McDonald, Gauge singlet scalar as inflaton and thermal relic dark matter, Phys. Rev. D80 (2009) 123507 [0909.0520]. 
[42] R. N. Lerner and J. McDonald, Higgs inflation and naturalness, JCAP 1004 (2010) 015 [0912.5463].

[43] D. Burns, S. Karamitsos and A. Pilaftsis, Frame-covariant formulation of inflation in scalar-curvature theories, Nucl. Phys. B907 (2016) 785 [1603.03730].

[44] A. Karam, T. Pappas and K. Tamvakis, Frame-dependence of higher-order inflationary observables in scalar-tensor theories, Phys. Rev. D96 (2017) 064036 [1707.00984].

[45] M. Postma and M. Volponi, Equivalence of the Einstein and Jordan frames, Phys. Rev. D90 (2014) 103516 [1407.6874].

[46] S. Ferrara, R. Kallosh, A. Linde and M. Porrati, Minimal supergravity models of inflation, Phys. Rev. D88 (2013) 085038 [1307.7696].

[47] R. Kallosh, A. Linde and D. Roest, Superconformal inflationary $\alpha$-attractors, JHEP 11 (2013) 198 [1311.0472].

[48] J. Goldstone, Field theories with superconductor solutions, Nuovo Cim. 19 (1961) 154.

[49] A. Vilenkin, Topological inflation, Phys. Rev. Lett. 72 (1994) 3137 [hep-th/9402085].

[50] A. D. Linde and D. A. Linde, Topological defects as seeds for eternal inflation, Phys. Rev. D50 (1994) 2456 [hep-th/9402115].

[51] C. Destri, H. J. de Vega and N. G. Sanchez, MCMC analysis of WMAP3 and SDSS data points to broken symmetry inflaton potentials and provides a lower bound on the tensor to scalar ratio, Phys. Rev. D77 (2008) 043509 [astro-ph/0703417].

[52] R. Kallosh and A. D. Linde, Testing string theory with CMB, JCAP 0704 (2007) 017 [0704.0647].

[53] T. L. Smith, M. Kamionkowski and A. Cooray, The inflationary gravitational-wave background and measurements of the scalar spectral index, Phys. Rev. D78 (2008) 083525 [0802.1530].

[54] M. U. Rehman, Q. Shafi and J. R. Wickman, GUT inflation and proton decay after WMAP5, Phys. Rev. D78 (2008) 123516 [0810.3625].

[55] N. Okada, V. N. Şenoğuz and Q. Shafi, The observational status of simple inflationary models: an update, Turk. J. Phys. 40 (2016) 150 [1403.6403].

[56] A. Ashoorioon and M. M. Sheikh-Jabbari, Gauged M-flation after BICEP2, Phys. Lett. B739 (2014) 391 [1405.1685].

[57] F. S. Accetta, D. J. Zoller and M. S. Turner, Induced gravity inflation, Phys. Rev. D31 (1985) 3046.

[58] D. I. Kaiser, Primordial spectral indices from generalized Einstein theories, Phys. Rev. D52 (1995) 4295 [astro-ph/9408044].

[59] A. Cerioni, F. Finelli, A. Tronconi and G. Venturi, Inflation and reheating in induced gravity, Phys. Lett. B681 (2009) 383 [0906.1902].

[60] A. Tronconi, Asymptotically safe non-minimal inflation, JCAP 1707 (2017) 015 [1704.05312].

[61] K. I. Izawa and T. Yanagida, Natural new inflation in broken supergravity, Phys. Lett. B393 (1997) 331 [hep-ph/9608359].

[62] M. Kawasaki, M. Yamaguchi and J. Yokoyama, Inflation with a running spectral index in supergravity, Phys. Rev. D68 (2003) 023508 [hep-ph/0304161].

[63] M. Yamaguchi and J. Yokoyama, Smooth hybrid inflation in supergravity with a running spectral index and early star formation, Phys. Rev. D70 (2004) 023513 [hep-ph/0402282].

[64] V. N. Şenoğuz and Q. Shafi, New inflation, preinflation, and leptogenesis, Phys. Lett. B596 (2004) 8 [hep-ph/0403294]. 
[65] D. Boyanovsky, H. J. de Vega, C. M. Ho and N. G. Sanchez, New inflation vs. chaotic inflation, higher degree potentials and the reconstruction program in light of WMAP3, Phys. Rev. D75 (2007) 123504 [astro-ph/0702627].

[66] C. Destri, H. J. de Vega and N. G. Sanchez, Higher order terms in the inflaton potential and the lower bound on the tensor to scalar ratio r, Annals Phys. 326 (2011) 578 [0906.4102].

[67] L. Boubekeur and D. H. Lyth, Hilltop inflation, JCAP 0507 (2005) 010 [hep-ph/0502047].

[68] S. R. Coleman and E. J. Weinberg, Radiative corrections as the origin of spontaneous symmetry breaking, Phys. Rev. D7 (1973) 1888.

[69] Q. Shafi and A. Vilenkin, Inflation with SU(5), Phys. Rev. Lett. 52 (1984) 691.

[70] A. Albrecht and R. H. Brandenberger, On the realization of new inflation, Phys. Rev. D31 (1985) 1225.

[71] A. D. Linde, Particle physics and inflationary cosmology, Contemp. Concepts Phys. 5 (1990) 1 [hep-th/0503203].

[72] Q. Shafi and V. N. Şenoğuz, Coleman-Weinberg potential in good agreement with WMAP, Phys. Rev. D73 (2006) 127301 [astro-ph/0603830].

[73] G. Barenboim, E. J. Chun and H. M. Lee, Coleman-Weinberg inflation in light of Planck, Phys. Lett. B730 (2014) 81 [1309.1695].

[74] K. Kannike, A. Racioppi and M. Raidal, Embedding inflation into the Standard Model - more evidence for classical scale invariance, JHEP 06 (2014) 154 [1405.3987].

[75] V. N. Şenoğuz and Q. Shafi, Primordial monopoles, proton decay, gravity waves and GUT inflation, Phys. Lett. B752 (2016) 169 [1510.04442].

[76] S. Iso, K. Kohri and K. Shimada, Small field Coleman-Weinberg inflation driven by a fermion condensate, Phys. Rev. D91 (2015) 044006 [1408.2339].

[77] K. Kaneta, O. Seto and R. Takahashi, Very low scale Coleman-Weinberg inflation with nonminimal coupling, Phys. Rev. D97 (2018) 063004 [1708.06455].

[78] G. Panotopoulos, Nonminimal GUT inflation after Planck results, Phys. Rev. D89 (2014) 047301 [1403.0931].

[79] L. Marzola, A. Racioppi, M. Raidal, F. R. Urban and H. Veermäe, Non-minimal CW inflation, electroweak symmetry breaking and the 750 GeV anomaly, JHEP 03 (2016) 190 [1512.09136].

[80] K. Kannike, A. Racioppi and M. Raidal, Linear inflation from quartic potential, JHEP 01 (2016) 035 [1509.05423].

[81] D. Baumann and L. McAllister, Inflation and string theory. Cambridge University Press, 2015, [1404.2601].

[82] T. Muta and S. D. Odintsov, Model dependence of the nonminimal scalar graviton effective coupling constant in curved space-time, Mod. Phys. Lett. A6 (1991) 3641.

[83] C. P. Burgess, H. M. Lee and M. Trott, Power-counting and the validity of the classical approximation during inflation, JHEP 09 (2009) 103 [0902.4465].

[84] M. P. Hertzberg, On inflation with non-minimal coupling, JHEP 11 (2010) 023 [1002.2995].

[85] F. Bezrukov, A. Magnin, M. Shaposhnikov and S. Sibiryakov, Higgs inflation: consistency and generalisations, JHEP 01 (2011) 016 [1008.5157]. 